\title{
Techniques for the detection of pathogenic Cryptococcus species in wood decay substrata and the evaluation of viability in stored samples
}

\author{
Christian Alvarez²/+, Glaucia Gonçalves Barbosa', Raquel de Vasconcellos Carvalhaes de Oliveira', \\ Bernardina Penarrieta Morales', Bodo Wanke', Márcia dos Santos Lazéra'
}

'Laboratório de Micologia, Instituto de Pesquisa Clínica Evandro Chagas-Fiocruz, Rio de Janeiro, RJ, Brasil

${ }^{2}$ Laboratorio de Micologia del Hospital del Niño Jesús, Tucumán, Argentina

In this study, we evaluated several techniques for the detection of the yeast form of Cryptococcus in decaying wood and measured the viability of these fungi in environmental samples stored in the laboratory. Samples were collected from a tree known to be positive for Cryptococcus and were each inoculated on 10 Niger seed agar (NSA) plates. The conventional technique (CT) yielded a greater number of positive samples and indicated a higher fungal density [in colony forming units per gram of wood $\left(C F U . g^{-1}\right)$ ] compared to the humid swab technique (ST). However, the difference in positive and false negative results between the CT-ST was not significant. The threshold of detec-

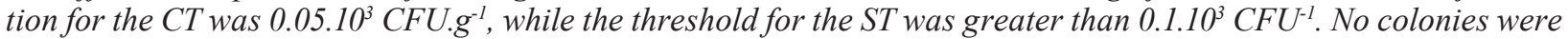
recovered using the dry swab technique. We also determined the viability of Cryptococcus in wood samples stored for 45 days at $25^{\circ} \mathrm{C}$ using the CT and ST and found that samples not only continued to yield a positive response, but also exhibited an increase in CFU. $\mathrm{g}^{-1}$, suggesting that Cryptococcus is able to grow in stored environmental samples. The ST.1, in which samples collected with swabs were immediately plated on NSA medium, was more efficient and less laborious than either the CT or ST and required approximately 10 min to perform; however, additional studies are needed to validate this technique.

Key words: isolation techniques - Cryptococcus neoformans - Cryptococcus gattii - wood decay substrata

Cryptococcosis is an emerging systemic mycosis worldwide and is the major cause of fungal meningoencephalitis. The causative agents of cryptococcosis are the zoopathogenic, basidiomycetous, phenol-oxidase-producing encapsulated yeasts belonging to the Cryptococcus neoformans/Cryptococcus gattii species complex; formerly considered subspecies, C. neoformans and C. gattii are now recognised as distinct species (Kwon-Chung et al. 2002). The primary ecologic niche of both pathogens is the decaying wood of trees and was first described by Lazéra et al. (2000). The wide spectrum of host trees and the non-specific relationship of these cryptococcal species with ligninaceous substrata have become increasingly evident through studies conducted in different countries (Lazéra et al. 1996, 2000, Fortes et al. 2001, Granados \& Castañeda 2005, Refojo et al. 2009, Chowdhary et al. 2012). The use of different or modified techniques to isolate Cryptococcus from the environment has made it difficult to compare studies among regions, habitats and specific sources of Cryptococcus. Environmental studies of C. neoformans and $C$. gattii have traditionally analysed wood scrapings using the conventional technique (CT) in which saline-

Financial support: IPEC-FIOCRUZ, FAPERJ (E-26/110.486.2007)

+ Corresponding author: bqco_chal@hotmail.com

Received 21 May 12

Accepted 20 August 12 suspended samples are plated on Niger seed (Guizotia abyssinica) extract agar (NSA), with some variations (Lazéra et al. 2000, Fortes et al. 2001, Kidd et al. 2004). In recent years, however, a variation on the conventional collection procedure known as the swabbing technique (ST) has been implemented, as well as other procedures that are similar to the CT (Davel et al. 2003, Randhawa et al. 2005, Kidd et al. 2007). The aim of this study was to compare the efficiency of these and other techniques for the isolation of C. neoformans and C. gattii from decomposing wood within the hollows of living trees and to evaluate the viability of these pathogens in stored environmental samples.

We obtained samples for this study from the inner surface of a hollow Cassia grandis tree that first tested positive for C. neoformans in 1996 (Lazéra et al. 1996) and was subsequently positive for $C$. neoformans and $C$. gattii at the time of this study in 2007 (B Morales et al., unpublished observations). Ten areas of approximately 25 $\mathrm{cm}^{2}$ each on the inner surface of the tree were marked for collection. Each sample analysed by each technique was plated on 10 NSA plates containing $200 \mathrm{mg} / \mathrm{L}$ chloramphenicol. Moist, shiny, dark brown colonies were identified as belonging to the C. neoformans/C. gattii species complex by morphophysiological tests. Positive samples were capsulated yeasts without pseudohyphae, thermotolerant at $37^{\circ} \mathrm{C}$ and cycloheximide-sensitive at $25^{\circ} \mathrm{C}$ and $37^{\circ} \mathrm{C}$ (Lazéra et al. 1996). Samples were further analysed using the bioMérieux Vitek 1-32 System to confirm that the isolates belonged to the C. neoformans/C. gattii species complex. No distinction between these two species was made in this study. 
To compare the two isolation techniques, samples were collected from each of the 10 areas on the same day. For the CT, samples were obtained by scraping, transported in sterile bottles at room temperature (RT) and processed within 2 h. Following vigorous homogenisation with a mortar, $1 \mathrm{~g}$ of the sample was suspended in $50 \mathrm{~mL}$ of sterile saline containing chloramphenicol (SSC) at 0.2 g.L $\mathrm{L}^{-1}$, shaken vigorously for $5 \mathrm{~min}$ and allowed to rest for $30 \mathrm{~min}$. From this settled suspension, $0.1 \mathrm{~mL}$ of supernatant was plated on NSA plates, which were then incubated at $25^{\circ} \mathrm{C}$ for 5 days.

For the ST, samples were collected from each area using swabs dampened with SSC, which were transported in a sterile paper bag at RT and processed within $2 \mathrm{~h}$. In the laboratory, the end of the swab was transferred to a plastic tube containing $10 \mathrm{~mL}$ of SSC and was shaken vigorously for $5 \mathrm{~min}$. The remaining processing was identical to that of the CT; for the dry swab technique (DST), samples were collected and processed as for the ST, but dry cotton-tipped swabs were used for sample collection.

The viability of $C$. neoformans/C. gattii cells in samples of decaying wood stored 45 days at RT was also analysed. The samples that were obtained in the initial CT experiment, which had been stored in sterile plastic containers, were reprocessed using the CT and ST; for the latter technique, the humid swab was placed in the plastic bag containing the sample and was processed as in the first experiment. The sample from area 6 was not reprocessed due to an insufficient amount of material.

Finally, the processing times were compared for the three techniques used to isolate cryptococcal species in this study. Samples were collected on the same day from positive and negative areas that were chosen based on the results of the initial CT experiment. The samples were collected and processed using the CT, ST and DST approaches as described above. In addition, we evaluated two variations of the ST and the DST. For ST.1 and DST.1, NSA plates were inoculated in the field with a humid or dry swab, respectively, and the plates were transported to the RT laboratory for subsequent observation and subcultivation of colonies consistent with the yeast form of Cryptococcus. For ST.2 and DST.2, the samples were collected and were processed as for the ST and DST after one week of storage at RT in a sterile paper bag.

In this study, the fungal burden in each positive area was estimated from the total number of colonies observed on the NSA plates and is expressed as colony forming units per gram of wood (CFU. $\left.\mathrm{g}^{-1}\right)$ for the CT and as colony forming units per swab (CFU/swab) for the ST, DST, ST.1, DST.1, ST.2 and DST.2.

The summary measures of the CFU data, including averages, standard deviations (SD), coefficients of variation and minimum and maximum values were submitted for statistical analysis. Fisher's exact test was used to compare the proportions of positive and negative results among the studied techniques. Microsoft Excel 2003 and the Statistical Package for Social Sciences version 16.0 were used for data processing and statistical analysis. p-values $<0.05$ indicated significant differences for the tests used. Differences in the number of colonies amongst the techniques and processing times were evaluated using the Wilcoxon unpaired test, which is equivalent to the Mann-Whitney test.

One hundred forty-two colonies identified as $C$. neoformans/C. gattii were isolated: $64.8 \%$ by the CT and $35.2 \%$ by the ST. The CT yielded a higher positivity by area $(5 / 10)$ and a greater number of colony forming units (92 CFU.g $\left.{ }^{-1}\right)$ compared to the ST (2/10 and $50 \mathrm{CFU} / \mathrm{swab}$, respectively); however, these differences were not significant. No colonies were observed in any samples analysed by the DST. At 5\% ( $p=0.1667$, Fisher's exact test), there was no significant difference between the CT-ST with respect to the number of positive and false negative results.

The mean and SD of the number of colonies isolated from each of the analysed areas was $18.4 \pm 24.05 \mathrm{CFU}^{-1} \mathrm{~g}^{-1}$ for the CT and $10 \pm 16.49 \mathrm{CFU} / \mathrm{swab}$ for the ST, with coefficients of variation of $130.69 \%$ and $164.92 \%$, respectively. The threshold of detection was $0.05 .10^{3} \mathrm{CFU} . \mathrm{g}^{-1}$ for the $\mathrm{CT}$ and $0.12 .10^{3} \mathrm{CFU} / \mathrm{swab}$ for the ST (Table). Comparing the results of the $\mathrm{CT}$ and the ST for the same sampling areas revealed that the ST was positive in areas with 1.800 CFU.g ${ }^{-1}$ or greater and was negative in areas with 50 or $100 \mathrm{CFU}^{-1} \mathrm{~g}^{-1}$, demonstrating that the dilution and volume of the original suspension are critical for a positive result, given the low density of these organisms.

In our analysis, we demonstrated that the CT was more efficient than the ST; however, this difference was not significant. Our results differ from those obtained by Randhawa et al. (2005) in India. In that study, which compared swabbing vs. the CT, the authors concluded that the ST was more efficient than the CT. However, a greater final dilution (1/100) and a smaller volume of the supernatant was plated on NSA plates $(0.1 \mathrm{~mL}$ each on 2 plates) for the CT, which lowered the threshold of detection to $0.5 .10^{3}$ CFU.g ${ }^{-1}$, compared to $0.05 .10^{3} \mathrm{CFU}_{\text {. }}{ }^{-1}$ in the present study. Therefore, slight differences in technique prevent a comparative analysis of the CT results in that study and those of the present study.

We also evaluated the viability of $C$. neoformans $/ C$. gattii following storage at RT for 45 days. The results were similar to those obtained with freshly isolated samples: the same proportion of areas tested positive with the CT (5/9) and the ST (2/9). A total of 2,022 colonies identified as $C$. neoformans/C. gattii were isolated: $90.2 \%$ by the CT and $9.8 \%$ by the ST. The greatest fungal burden observed was $8.96 .10^{4} \mathrm{CFU}^{-1}$ for sample A5 with the CT; when freshly isolated samples were used, sample A3 yielded the greatest fungal burden $\left(2.6 .10^{3} \mathrm{CFU} \cdot \mathrm{g}^{-1}\right)$. The mean and SD of the number of colonies isolated from each of the analysed areas was $364.6 \pm 798$ CFU.g $^{-1}$ for the CT and $99.5 \pm 61.52 \mathrm{CFU} /$ swab for the ST, with coefficients of variation of $218.87 \%$ and $157.24 \%$, respectively (Table). The threshold of detection was $0.05 .10^{3}$ CFU.g ${ }^{-1}$ for the CT and $0.56 .10^{3} \mathrm{CFU} /$ swab for the ST. However, there was no significant difference between the CT-ST $(\mathrm{p}=0.1667)$.

We observed no loss of $C$. neoformans/C. gattii viability in decaying wood samples following storage. On the contrary, a greater number of CFU was obtained from the wood substrata that were stored for 45 days than from the freshly isolated samples, suggesting active growth of C. neoformans/C. gattii. It must be emphasised that the 


\section{TABLE}

Results obtained by the conventional technique (CT) and swabbing technique (ST) in the two first experiment of the hollow of Cassia grandis informed positive for Cryptococcus neoformans by Lazéra et al. (1996)

\begin{tabular}{|c|c|c|c|c|}
\hline & \multicolumn{2}{|c|}{ First experiment } & \multicolumn{2}{|c|}{ Second experiment } \\
\hline & $\mathrm{CT}$ & ST & $\mathrm{CT}$ & ST \\
\hline Areas tested (n) & 10 & 10 & 9 & 9 \\
\hline Areas positive $[\mathrm{n}(\%)]$ & $5(50)$ & $2(20)$ & $5(56)$ & $2(22.2)$ \\
\hline False negative $[\mathrm{n}(\%)]$ & $0(0)$ & $3(30)$ & $0(0)$ & $3(33.3)$ \\
\hline Threshold of detection express for the CT and CFU/swab for the ST (CFU.g $\mathrm{g}^{-1}$ ) & $0.05 .10^{3}$ & $0.12 .10^{3}$ & $0.05 .10^{3}$ & $0.56 .10^{3}$ \\
\hline Mean \pm SD detection express for the CT and CFU/swab for the ST (CFU.g $\left.{ }^{-1}\right)$ & $18.4 \pm 24.05$ & $10 \pm 16.49$ & $364.6 \pm 798$ & $99.5 \pm 61.52$ \\
\hline Coefficients of variation $(\%)$ & 130.69 & 164.92 & 218.87 & 157.24 \\
\hline
\end{tabular}

CFU: colony forming units; SD: standard deviation.

stored samples were not treated to eliminate other microorganisms (bacterial and fungal filaments) that may compete with Cryptococcus for nutrients. The ability of stored samples to maintain viability may be particularly useful for field studies located far from the laboratory.

In addition to evaluating multiple collection techniques and the long-term viability of $C$. neoformans $/ C$. gattii, we compared the processing times for samples collected using the techniques described above. Two hundred third-five colonies of $C$. neoformans $/ C$. gattii were isolated from positive areas as follows: $65.1 \%$ with the ST.1, 22.1\% with the CT, $12.3 \%$ with the ST and $0.5 \%$ with the DST.1. None of the other procedures yielded colonies. Statistical analysis revealed a significant difference (at the 5\% level) in the CFU obtained with each technique. The ST.1 was more efficient than the CT (p $=0.0127)$ and the ST $(\mathrm{p}=0.0017)$ and the CT was more efficient than the ST $(p=0.0280)$.

In this study, the number of positive results among dampened samples decreased rapidly due to bacterial growth as the time between sampling and processing increased. In addition, the $\mathrm{CT}$ is a more laborious technique than either the ST.1 or the ST. It was easier to collect wood samples from inside the cavity with a swab and the processing time for the ST.1 was approximately 10 min, compared with approximately $2 \mathrm{~h}$ with the CT. Additionally, the ST.1 reduced the amount of sample manipulation and the time of exposure to potentially pathogenic Cryptococcus species. The disadvantage of using the ST.1, however, is that the laboratory must be near the site of collection; after two-three days of incubation on NSA medium at $25^{\circ} \mathrm{C}$, it is difficult to identify and subculture the dark brown colonies characteristic of Cryptococcus due to the growth of bacterial and fungal filaments.

The results of our study support the idea that the occurrence of Cryptococcus species in the hollows of trees is microfocal as areas of the tree that tested negative were adjacent to areas that tested positive. Thus, a low number of samples from a certain microenvironment may test negative despite the presence of Cryptococcus. Two additional factors that affect the detection of Cryptococcus in the environment are the efficiency of the collection technique and the concentration of fungal cells present in the sample collected. Increasing the number of CFU analysed increases the probability of isolating $C$. neoformans and $C$. gattii, as well as identifying isolates with different genotypes, which in turn expands our knowledge of Cryptococcus ecology and population structure.

We conclude that the CT is the most efficient technique for the detection of $C$. neoformans and $C$. gattii in decaying wood. However, considering sample processing time, the rapid plating of samples with a swab (ST.1) was more efficient than the CT. This result was based on one positive area with a high density of Cryptococcus and should be evaluated further in additional comparative studies. For studies with a large number of samples, the ST may be a useful tool, but a decrease in positive results from substrata with low fungal density must be expected. We have found that, for the investigation of environmental sources of cryptococcosis agents, the best technique is to collect samples by scraping and store these samples for processing. Sampling should be performed with the humid swab technique and the samples should either be processed in the laboratory, as in the ST, or plated immediately on NSA medium as in the ST.1. If either the ST or the ST.1 is negative for dark brown colonies, the samples should be processed again using the CT and the stored material.

\section{REFERENCES}

Chowdhary A, Randhawa HS, Prakash A, Méis JF 2012. Environmental prevalence of Cryptococcus neoformans and Cryptococcus gattii in India: an update. Crit Rev Microbiol 38: 1-16.

Davel G, Abrantes R, Brudny M, Córdoba S, Rodero L, Canteros CE, Perrotta D 2003. Primer aislamiento ambiental de Cryptococcus neoformans var. gattii en Argentina. Rev Arg Microbiol 35: $110-112$.

Fortes ST, Lazéra MS, Nishikawa MM, Macedo RCL, Wanke B 2001. First isolation of Cryptococcus neoformans var. gattii from native jungle tree in the Brazilian Amazon rainforest. $M y$ coses 44: 137-140.

Granados DP, Castañeda E 2005. Isolation and characterization of Cryptococcus neoformans varieties recovered from natural 
sources in Bogotá, Colombia, and study of ecological conditions in the area. Microb Ecol 49: 282-290.

Kidd SE, Chow Y, Mak S, Bach PJ, Chen H, Hingston AO, Kronstad JW, Barlett KH 2007. Characterization of environmental sources of the human and animal pathogen Cryptococcus gattii in British Columbia, Canada, and the Pacific Northwest of the United States. Appl Environ Microbiol 73: 1433-1443.

Kidd SE, Hagen F, Tscharke RL, Huynh M, Bartlett KH, Fyfe M, MacDougall L, Boekhout T, Kwon-Chung KJ, Meyer W 2004. A rare genotype of Cryptococcus gattii caused the cryptococcosis outbreak on Vancouver Island (British Columbia, Canada). Proc Natl Acad Sci USA 101: 17258-17263.

Kwon-Chung KJ, Boekhout T, Fell JW, Diaz M 2002. Proposal to conserve the name Cryptococcus gattii against $C$. hondurianus and C. Bacillisporus (Basidiomycota, Hymenomycetes, Tremellomycetidae). Taxon 51: 804-806.
Lazéra MS, Cavalcanti MAS, Londero AT, Trilles L, Nishikawa MM, Wanke B 2000. Possible primary ecological niche of Cryptococcus neoformans. Med Mycol 38: 379-383.

Lazéra MS, Pires FDA, Camillo-Coura L, Nishikawa MM, Bezerra CCF, Trilles L, Wanke B 1996. Natural habitat of Cryptococcus neoformans var. neoformans in decaying wood forming hollows in living trees. J Med Vet Mycol 34: 127-131.

Randhawa HS, Kowshik T, Khan ZU 2005. Efficacy of swabbing versus a conventional technique for isolation of Cryptococcus neoformans from decayed wood in tree trunk hollows. Med Mycol 43: 67-71.

Refojo N, Perrota NDE, Brudny M, Abrantes R, Hevia AI, Davel G 2009. Isolation of Cryptococcus neoformans and Cryptococcus gattii from trunk hollows in living trees in Buenos Aires city, Argentina. Med Mycol 47: 177-184. 